\title{
An Analysis of Foot and Ankle Device Recalls by the Food and Drug Administration
}

\author{
Carl Pellerin ${ }^{1}$, Vinod Panchbhavi ${ }^{2}$, Cory F. Janney ${ }^{3}$ \\ 1. Department of Orthopaedic Surgery and Rehabilitation, University of Texas Medical, Galveston, USA 2. Department \\ of Orthopedics Surgery and Rehabilitation, University of Texas Medical Branch, Galveston, USA 3. Department of \\ Orthopedics, Naval Medical Center, San Diego, USA
}

Corresponding author: Cory F. Janney, janneyc@gmail.com

\begin{abstract}
\section{Introduction}

Orthopaedic devices represent $12 \%$ of all medical device recalls. Products are approved through pre-market approval (PMA) or the $510(\mathrm{k})$ premarket notification process. No previous evaluation was found in the literature evaluating foot and ankle device recalls. The field of foot and ankle subspecialty has seen a rapid growth in innovation related to implants in recent years.
\end{abstract}

\section{Methods}

The Food and Drug Administration (FDA) Device Recall database was evaluated for all foot and ankle devices from 2007 through 2017 for the manufacturer, process of approval, type of implant, recall class, dates of initiation and termination of the recall, manufacturer determined reason, quantity affected, and distribution within the United States or internationally.

\section{Results}

A total of 161 products from 33 companies were identified with 158 (98.1\%) approved through the 510(k) process. The most common reason for device recall was due to the device breaking intraoperatively or postoperatively. The average length of the recall was 487.5 days.

\section{Conclusions}

Device recall is not an uncommon event with the majority of products approved through the less demanding $510(\mathrm{k})$ process.

Received 07/25/2018 Review began $07 / 27 / 2018$ Review ended 08/03/2018 Published 08/09/2018

๑ Copyright 2018 Pellerin et al. This is an open access article distributed under the terms of the Creative Commons Attribution License CC-BY 3.0., which permits unrestricted use, distribution, and reproduction in any medium, provided the original author and source are credited.
Categories: Orthopedics, Quality Improvement, Healthcare Technology

Keywords: foot, ankle, fda, device, recall

\section{Introduction}

Medical device recalls are common. According to the U.S. Food and Drug Administration (FDA), there were 20,093 recalls instituted by 1,641 manufacturers between November 2002 and December 2012 [1]. The incidence of recalls has not decreased over time. This unfortunate circumstance was confirmed as the FDA implemented 13,937 additional device recalls from 2013 through 2017. Of these additional product recalls, 3,202 occurred in 2017 alone [2].

The FDA is responsible for overseeing and regulating medical devices within the United States. Medical devices are typically approved through two methods: the premarket approval (PMA) process and the 510(k) premarket notification process. The PMA process has proven to be more expensive and time-consuming as it requires clinical evidence for authorization [3]. On the other hand, the $510(\mathrm{k})$ premarket notification is an accelerated process that omits medical devices from the clinical trial requirements as long as the device is "substantially equivalent" to an alternatively utilized medical device [4]. Defective medical devices have been recalled after gaining prior FDA authorization regardless of approval process utilized.

The United States medical device market has attracted a large sum of wealth and expenses throughout the years. In fact, this market was estimated to be over $\$ 133$ billion in 2016 [5]. The economic impact for the numerous categories of medical devices varies significantly [6]. In the field of orthopaedics, the majority of medical devices are utilized for procedures such as joint replacement (projected U.S. market of \$10.3 billion in 2018) and fracture management (projected U.S. market of $\$ 4.3$ billion in 2015) [3, 7-8].

Although various medical devices have been utilized in operating rooms throughout the years, there is limited research evaluating the rationalization behind orthopaedic device recalls. Orthopaedic devices represent $12 \%$ of all medical device recalls [9]. Yet, no previous study was found in the literature examining foot and ankle devices with regards to recalls. Thus, this paper aims to compile and evaluate the reasons behind why ankle and foot orthopaedic devices have been recalled between 1 January 2007 through 31 December 2017. We hypothesized that products approved through the 510(k) approval process would have a higher recall rate. 


\section{Cureus}

\section{Materials And Methods}

The Food and Drug Administration Device Recall database was queried for all foot and ankle devices from 1 January 2007 through 31 December 2017 by searching for the following terms: foot, ankle, toe, tibiotalar, subtalar, talus, calcaneus, navicular, cuboid, cuneiform, metatarsal, and phalanx [10]. Duplicates were removed from the search. The products were then verified as foot and ankle devices which may be used by a foot and ankle orthopaedic surgeon or podiatrist. Each recall was then used to evaluate the manufacturer, type of implant, recall class, date of initiation of the recall, date of termination of the recall, manufacturer determined reason, FDA determined reason, quantity affected, submission type, and the distribution within the United States or internationally.

\section{Results}

A total of 161 products from 33 different companies were identified (Table 1). The most common reason products were recalled was due to the device breaking intraoperatively or postoperatively, with one company recalling 78,594 suture anchors due to fractures of the hardware requiring revision surgery. Device breakage accounted for 39 of the 161 medical device recalls of implants or devices used during surgery with

12 occurring interoperatively and 26 postoperatively. One product was a prosthetic foot that could break under normal usage causing compromise to stability and function. The second-most common reason for recall was sterility concerns, which accounted for 37 recalls. Labeling issues and device design issues were also common reasons for recall, accounting for 30 and 27 recalls respectively. Employee error caused the least amount of recalls, with only one product removed from the market (Figure 1).

\begin{tabular}{|l|l|}
\hline Recall reason & Number of products recalled \\
\hline Product breakage & 39 \\
\hline Sterility issue & 37 \\
Labeling design & 30 \\
\hline Device design & 27 \\
Manufacturing defect & 22 \\
Software design & 3 \\
\hline Packaging issue & 2 \\
\hline Employee error & 1 \\
\hline Total products & 161 \\
\hline
\end{tabular}

TABLE 1: Manufacturer-determined reasoning for recall of the devices from January 1, 2007 through December 31, 2017. 


\section{Cureus}

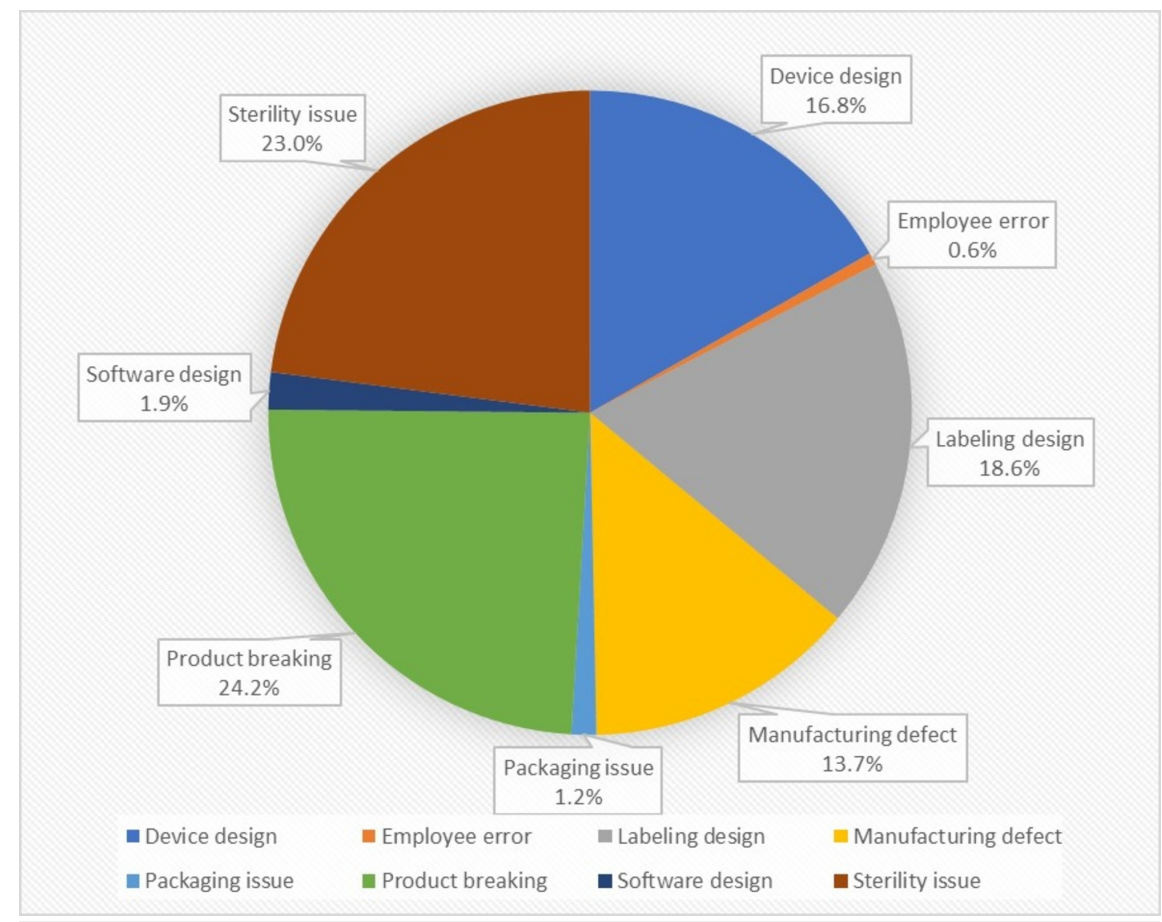

FIGURE 1: Manufacturer-determined reasoning for all products recalled.

The average length of the active recall time from initiation to termination was 487.5 days. The smallest number of recalled products in commerce were two sizes of Mitek SpiraLok anchors (DePuy Mitek Inc., Raynham, Massachusetts, US) with zero distributed that were recalled secondary to concerns for hardware failure. The largest number of recalled products were Synthes screws (Synthes Products LLC, West Chester, Pennsylvania) (cancellous, cortical, and cancellous of various sizes) with 19,497,844 screws recalled due to labeling issues.

Of the 161 medical devices that were recalled, 158 (98.1\%) were approved through the 510(k) premarket notification process. Conversely, only one product was authorized through the premarket approval (PMA) process. The submission procedure was not indicated for the remaining two medical devices (Figure 2).

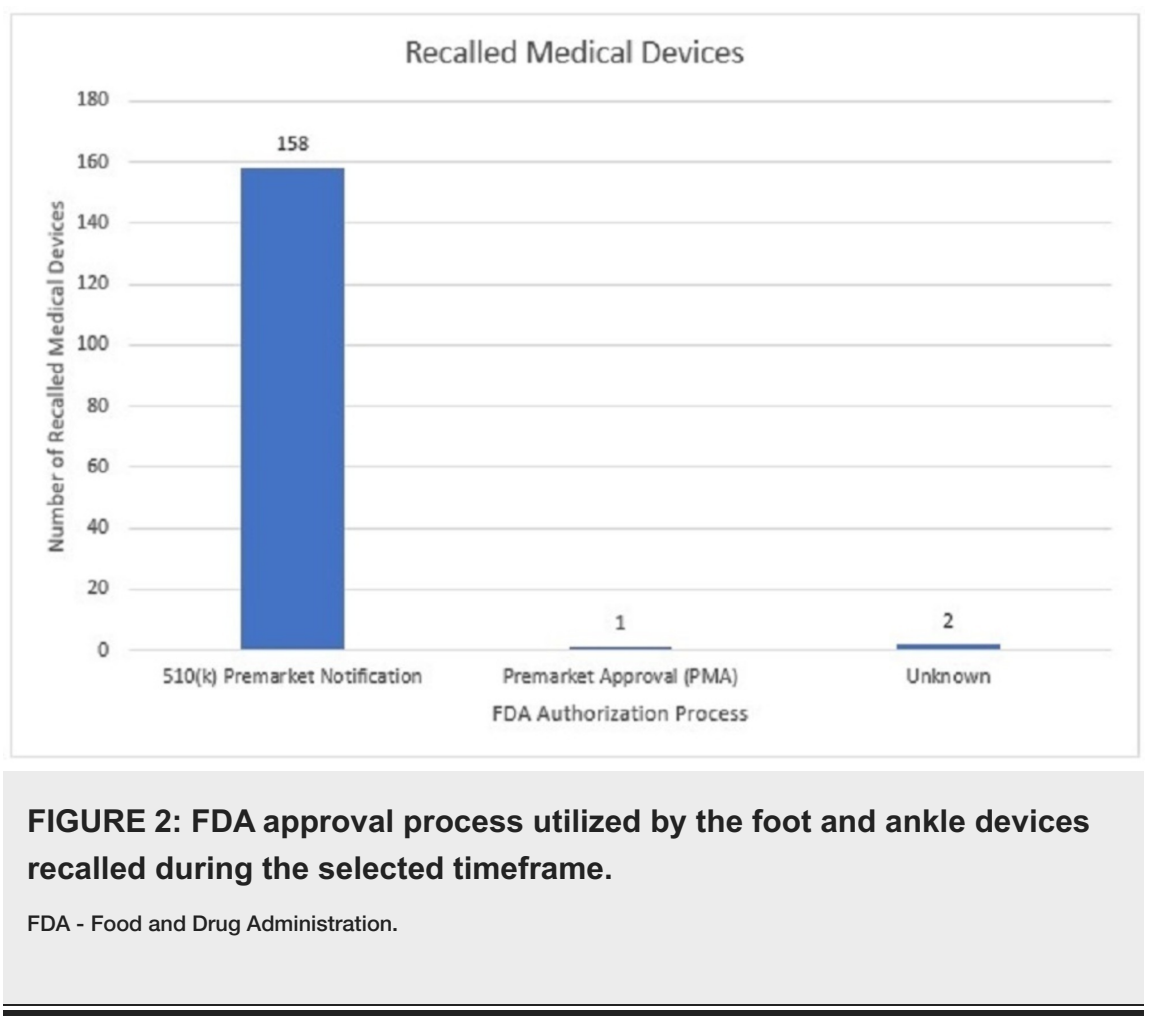




\section{Discussion}

During the recall process, it is the manufacturers' duty to notify surgeons. The surgeons should then identify the patients affected and notify them, making sure they understand the reasons for the recall as well as what the risks are to the patient [11]. Although the surgeon may be concerned about litigation, the cases are categorized as product liability cases and generally are not considered the surgeon's fault [12]. These patients should be followed frequently but prophylactic surgery is generally not recommended [13].

The overwhelming majority of recalls involved products approved through the $510(\mathrm{k})$ premarket notification process. This process does not involve the same stringent standards as the PMA application process. The PMA process requires clinical evidence before approval is granted, often requiring evidence on the level of randomized clinical trials or prospective data compared with historical controls [3-4, 14]. This study further supports the idea that products approved through the PMA process are less likely to be recalled $[1,15]$. There is a lack of clinical data with products approved through the $510(\mathrm{k})$ process that may cause the devices to carry a greater risk to patients. With regards to total hip arthroplasty implants, it is estimated that only $15 \%$ of these devices had data on clinical effectiveness published [16].

The 510(k) process allows a company to fast-track approval of a device by claiming it "substantially equivalent” to a device that has already been approved and marketed. Claiming that one device is equivalent to another device may be illogical even when the predicate comes from the same company [17-18]. This process may also lead to devices being approved on the predicate from another device that was itself approved through the $510(\mathrm{k})$ process, sometimes leading to decades between the original device and subsequent predicate devices without an increase in evidence of the product working [17].

Products breaking and sterility concerns were the most likely reasons for recall. Issues with products breaking intraoperatively or postoperatively may have been determined previously if the products had undergone the more demanding PMA process for approval. Sterility issues are often related to packaging which may not have been determined through a different testing protocol.

This study does have several limitations. We focused only on implants utilized within foot and ankle orthopaedic surgery by attempting to identify these products within the FDA database by searching for a wide array of terms within the database. If a product did not register one of the keywords we utilized, we may have missed some of the devices that were recalled. We also were unable to identify the approval process for two devices: the SYMBIONIC LEG (Ossur Americas Inc., Foothill Ranch, California, US) and Centurion Sterile Rubber Bands (\#84 Rubber Bands Reorder EB84, Centurion Medical Products Corporation, Howell, Michigan, US). The SYMBIONIC LEG is an integrated prosthetic leg for transfemoral and knee disarticulations that was recalled due to a software design error that failed to notify patients of a low battery before shutting down. The Centurion Sterile Rubber Bands were mislabeled as "latex free" erroneously. These rubber bands did, in fact, contain natural latex rubber and were recalled to prevent patients from having an allergic reaction.

Another limitation is that although the FDA-determined reasons were provided in the database, this may not correlate exactly with the manufacturer's reason for initiating a recall. Therefore, we chose to focus on the manufacturer's reasoning for initiating the recall. We were also unable to ascertain the costs associated with the initiation of these recalls or the direct effect on patients as this data is not readily available within the database.

\section{Conclusions}

A large proportion of implants used for foot and ankle surgery approved through 501(k) process were recalled when compared to the proportion of implants approved through the more stringent PMA process. Surgeons should stay abreast of recalls amongst the products that they use frequently. Although recalls are not often considered to be the fault of the operating surgeon, the patient's best interest is served with close follow up and making sure the patients is knowledgeable regarding the issues associated with the products themselves. Streamlining the PMA process or changing the $510(\mathrm{k})$ process may enable manufacturers to improve upon the safety of their devices.

\section{Additional Information \\ Disclosures}

Human subjects: Consent was obtained by all participants in this study. Animal subjects: All authors have confirmed that this study did not involve animal subjects or tissue. Conflicts of interest: In compliance with the ICMJE uniform disclosure form, all authors declare the following: Payment/services info: All authors have declared that no financial support was received from any organization for the submitted work. Financial relationships: Vinod Panchbhavi declare(s) personal fees from Stryker. Vinod Panchbhavi declare(s) Editor for a Journal from Lippincott Williams \& Wilkins (. Editor for Techniques in Foot \& Ankle Surgery. Other relationships: The views expressed in this article are those of the author(s) and do not necessarily reflect the official policy or position of the Department of the Navy, Department of Defense, or the United States Government. LCDR Cory Janney is a military service member (or employee of the U.S. Government). This work was prepared as part of his official duties. Title 17, USC, §105 provides that 'Copyright protection under this title is not available for any work of the U.S. Government.' Title 17, USC, $\S 101$ defines a U.S. Government work as a work prepared by a military service member or employee of the 


\section{References}

1. Day CS, Park DJ, Rozenshteyn FS, Owusu-Sarpong N, Gonzalez A: Analysis of FDA-approved orthopaedic devices and their recalls. J Bone Joint Surg Am. 2016, 98:517-524. 10.2106/JBJS.15.00286

2. Device recalls in 2017: making sense of the numbers . (2018). Accessed: June 18, 2018: https://www.raps.org/regulatory-focus\%E2\%84\%A2/news-articles/2018/1/device-recalls-in-2017-makingsense-of-the-numbers.

3. Yang BW, Iorio ML, Day CS: Orthopaedic device approval through the premarket approval process: a financial feasibility analysis for a single center. J Bone Joint Surg Am. 2017, 99:26. 10.2106/JBJS.16.00050

4. U.S. Food and Drug Administration: overview of device regulation . (2018). Accessed: June 18, 2018: http://www.fda.gov/MedicalDevices/DeviceRegulationandGuidance/Overview/.

5. Medical technology spotlight: the medical technology industry in the United States . Accessed: June 18, 2018: https://www.selectusa.gov/medical-device-industry-united-states .

6. ORTHOKNOW: strategic insights into the orthopaedic industry (2012) . (2012). Accessed: June 18, 2018 : https://www.orthoworld.com/index.php/fileproc/index/knowentADJADJorthoknowADJADJ2012ADJADJorthoknow1206LXLXL

7. U.S. markets for fracture fixation products. (2011). Accessed: June 18, 2018: https://sintel.com/marketreports-page.php?id=A326.

8. Global market for joint reconstruction and replacement to reach $\$ 16.2$ billion in 2018 . (2014). Accessed: June 18, 2018: https://www.bccresearch.com/pressroom/hlc/global-market-for-joint-reconstruction-andreplacement-to-reach-\$16.2-billi....

9. Medical devices: FDA should enhance its oversight of recalls . (2011). Accessed: June 18, 2018: https://www.gao.gov/new.items/d11468.pdf.

10. U.S. Food and Drug Administration: medical device recalls. (2018). Accessed: June 18, 2018: https://www.accessdata.fda.gov/scripts/cdrh/cfdocs/cfRES/res.cfm.

11. Racine J: Orthopedic medical devices: ethical questions, implant recalls and responsibility. R I Med J. 2013, 96:16-19. Accessed: August 6, 2018: http://www.rimed.org/rimedicaljournal/2013/06/2013-06-16-guestcommentary.pdf.

12. Weinberg J: Johnson \& Johnson is facing more than 10,000 lawsuits over an artificial hip that has been recalled. Rev Recent Clin Trials. 2013, 8:1. 10.2174/1574887111308010001

13. Pivec R, Meneghini RM, Hozack WJ, Westrich GH, Mont MA: Modular taper junction corrosion and failure: how to approach a recalled total hip arthroplasty implant. J Arthroplasty. 2014, 29:1-6. 10.1016/j.arth.2013.08.026

14. Fargen KM, Frei D, Fiorella D, McDougall CG, Myers PM, Hirsch JA, Mocco J: The FDA approval process for medical devices: an inherently flawed system or a valuable pathway for innovation?. J Neurointerv Surg. 2013, 5:269-275. 10.1136/neurintsurg-2012-010400

15. Zuckerman DM, Brown P, Nissen SE: Medical device recalls and the FDA approval process . Arch Intern Med. 2011, 171:1006-1011. 10.1001/archinternmed.2011.30

16. Mahomed NN, Syed K, Sledge CB, Brennan TA, Liang MH: Improving the postmarket surveillance of total joint arthroplasty devices. Open Rheumatol J. 2008, 2:7-12. 10.2174/1874312900802010007

17. Van Norman GA: Drugs, devices, and the FDA: Part 2: an overview of approval processes: FDA approval of medical devices. JACC Basic Transl Sci. 2016, 1:277-287. 10.1016/j.jacbts.2016.03.009

18. Naghshineh N, Brown S, Cederna PS, et al.: Demystifying the U.S. Food and Drug Administration: understanding regulatory pathways. Plast Reconstr Surg. 2014, 134:559-569.

10.1097/PRS.0000000000000477 